\title{
Correction \\ Correction: Phase I clinical study of the recombinant antibody-toxin ScFv(FRP5)-ETA specific for the ErbB2/HER2 receptor in patients with advanced solid malignomas
}

\author{
Gunter von Minckwitz¹, Sebastian Harder², Sascha Hövelmann ${ }^{3}$, Elke Jäger ${ }^{4}$, Salah-Eddin Al-Batran ${ }^{4}$, \\ Sibylle Loibl ${ }^{1}$, Akin Atmaca ${ }^{4}$, Christian Cimpoiasu ${ }^{1}$, Antje Neumann ${ }^{4}$, Aklil Abera ${ }^{3}$, Alexander Knuth ${ }^{4,5}$, \\ Manfred Kaufmann ${ }^{1}$, Dirk Jäger ${ }^{4,5}$, Alexander B Maurer ${ }^{3}$ and Winfried S Wels ${ }^{6}$
}

\author{
1Department of Gynecology and Obstetrics, University Hospital Frankfurt, Frankfurt am Main, Germany \\ 2Institute for Clinical Pharmacology, University Hospital Frankfurt, Frankfurt am Main, Germany \\ ${ }^{3}$ G2M Cancer Drugs AG, Frankfurt am Main, Germany \\ ${ }^{4}$ Medizinische Klinik II, Hämatologie-Onkologie, Krankenhaus Nordwest, Frankfurt am Main, Germany \\ ${ }^{5}$ Department of Oncology, University Hospital Zürich, Switzerland \\ ${ }^{6}$ Chemotherapeutisches Forschungsinstitut Georg-Speyer-Haus, Paul-Ehrlich-Straße 42-44, D-60596 Frankfurt am Main, Germany
}

Corresponding author: Winfried S Wels, wels@em.uni-frankfurt.de

Published: 24 February 2006

This article is online at http://breast-cancer-research.com/content/8/1/403

(c) 2006 BioMed Central Ltd

Following publication of the data presented by von Minckwitz and colleagues [1] it has been brought to our attention that some patients should be scored differently. Stable disease was seen in three of the eighteen patients instead of two of the eighteen patients: one patient with transitional cell carcinoma treated at $4 \mu \mathrm{g} / \mathrm{kg} \mathrm{scFv}$ (FRP5)-ETA per day, and two breast cancer patients treated at 4 and $12.5 \mu \mathrm{g} / \mathrm{kg}$ scFv(FRP5)-ETA per day. Disease progression occured in 9 of the eighteen patients evaluated (see corrected Table 2 overleaf). This does not affect the conclusions of our study. In addition we would like to correct the following errors: patient IDs for patients U01 and U02 in the original Table 2 were interchanged. In addition, patient $\mathrm{NO} 3$ had a grade 3 elevation of gamma-glutamyl transferase, and not grade 2 (see corrected Table 2 overleaf).

\section{Reference}

1. von Minckwitz G, Harder S, Hövelmann S, Jäger E, Al-Batran SE, Loibl S, Atmaca A, Cimpoiasu C, Neumann A, Abera A et al: Phase I clinical study of the recombinant antibody-toxin scFv(FRP5)-ETA specific for the ErbB2/HER2 receptor in patients with advanced solid malignomas. Breast Cancer Res 2005, 7:R617-R626. 
Table 2

\begin{tabular}{|c|c|c|c|c|c|c|}
\hline Patient & $\begin{array}{c}\text { Dose level } \\
(\mu \mathrm{g} / \mathrm{kg})\end{array}$ & Course of therapy & Toxicities $\geq$ grade 1 & $\begin{array}{l}\text { Dose-limiting } \\
\text { toxicity }\end{array}$ & $\begin{array}{l}\text { Neutralizing } \\
\text { antibodies }\end{array}$ & $\begin{array}{l}\text { Clinical } \\
\text { response }\end{array}$ \\
\hline N01 & 2 & According to plan & GGT grade 2 & No & No & Progression \\
\hline U01 & 2 & Stopped on day 10 & Cholestasis due to liver metastasis ${ }^{a}$ & No & n.d. & n.d. \\
\hline U02 & 2 & According to plan & None & No & n.d. & Progression \\
\hline N03 & 4 & According to plan & GGT grade 2 & No & No & Stable disease \\
\hline N04 & 4 & According to plan & ALT grade 1 & No & No & Stable disease \\
\hline N05 & 4 & According to plan & Hemoglobin grade $3^{a}$ & No & No & Progression \\
\hline N06 & 10 & According to plan & ALT grade 2, AST grade 1 & No & + & Progression \\
\hline N07 & 10 & According to plan & ALT/AST grade 1, GGT grade 3 & No & No & Progression \\
\hline U03 & 10 & According to plan & Fever and dyspnoe ${ }^{b}$ & No & ++ & n.d.c \\
\hline N13 & 12.5 & According to plan & ALT grade 1, GGT grade 2, AP grade 1 & No & No & Progression \\
\hline N14 & 12.5 & Stopped on day 8 & ALT/AST grade 3, GGT grade 2, LDH grade 1 & Yes & n.d. & n.d. \\
\hline N15 & 12.5 & According to plan & ALT grade 2, AST grade 1, AP grade 2 & No & + & Progression \\
\hline N17 & 12.5 & According to plan & ALT/AST grade 2 & No & No & Progression \\
\hline U04 & 12.5 & According to plan & Dyspnoe & No & No & n.d.c ${ }^{c}$ \\
\hline U05 & 12.5 & According to plan & None & No & ++ & Stable disease \\
\hline N09 & 20 & According to plan & ALT/AST grade 2 & No & +++ & Progression \\
\hline N10 & 20 & Stopped on day 8 & ALT grade 4, AST grade 3, GGT grade 2 & Yes & n.d. & n.d. \\
\hline N12 & 20 & Stopped on day 8 & ALT grade 3, AST grade 2 & Yes & n.d. & n.d.c ${ }^{c}$ \\
\hline
\end{tabular}

ALT, alanine aminotransferase; AST, aspartate aminotransferase; GGT, gamma-glutamyl transferase; n.d., not determined. ${ }^{a}$ Causal relationship with study drug unlikely.

bPatient U03 developed fever and dyspnoe after therapy on day 23 , which was resolved with antibiotics; the patient died on day 40 , causal relationship with study drug unlikely.

${ }^{c}$ Clinical signs of activity while on therapy including healing of cutaneous lesion (U03, U04), size reduction of lymph node metastasis (U03), and inflammatory response and softening of large tumor mass (N12). 